\title{
Optimized Common Parameter Set Extraction Framework by Multiple Benchmarking Applications on a Big Data Platform
}

\author{
Jongyeop Kim ${ }^{1, *}$, Abhilash Kancharla ${ }^{2}$, Jongho Seol ${ }^{2}$, Indy N. Park ${ }^{3}$, Nohpill Park ${ }^{2}$ \\ ${ }^{1}$ Math and Computer Science, Southern Arkansas University, Magnolia, AR 71753, USA \\ ${ }^{2}$ Computer Science, Oklahoma State University, Stillwater, OK USA \\ ${ }^{3}$ Computer Science, Oklahoma City University, Oklahoma City, OK, USA
}

ARTICLE INFO
Article History
Received 27 June 2018
Accepted 20 September 2018
Keywords
Big data
Hadoop
configuration
performance tuning

\section{ARTICLE INFO}

Received 27 June 2018

Keywords

performance tuning 


\section{PRELIMINARY}

In this section, the background knowledge of the Hadoop configuration parameters, parameter domains, couple of benchmarking techniques and several proposed algorithms will be reviewed.

\subsection{Hadoop Parameters}

The most important and fundamental concept of Hadoop is to reduce the overall processing time by distributing one task to multiple cluster nodes. To control the efficient operation between master and slave nodes [13], tuning of configuration parameter according to system environment is needed. Well-tuned parameter values will keep the system in optimal condition and ultimately improve system performance.

Apache Hadoop has more than 80 parameters [14] and 25 of these parameters are significantly affected map and reduce performance [4]. Those parameters are defined at the \$hadoop_home/conf/mapredsite.xml as one of the properties. For example, io.sort.mb with default value 100 and io.sort.record.percent with value 0.05 can be described as follows:

- Configuration for mapred-site.xml

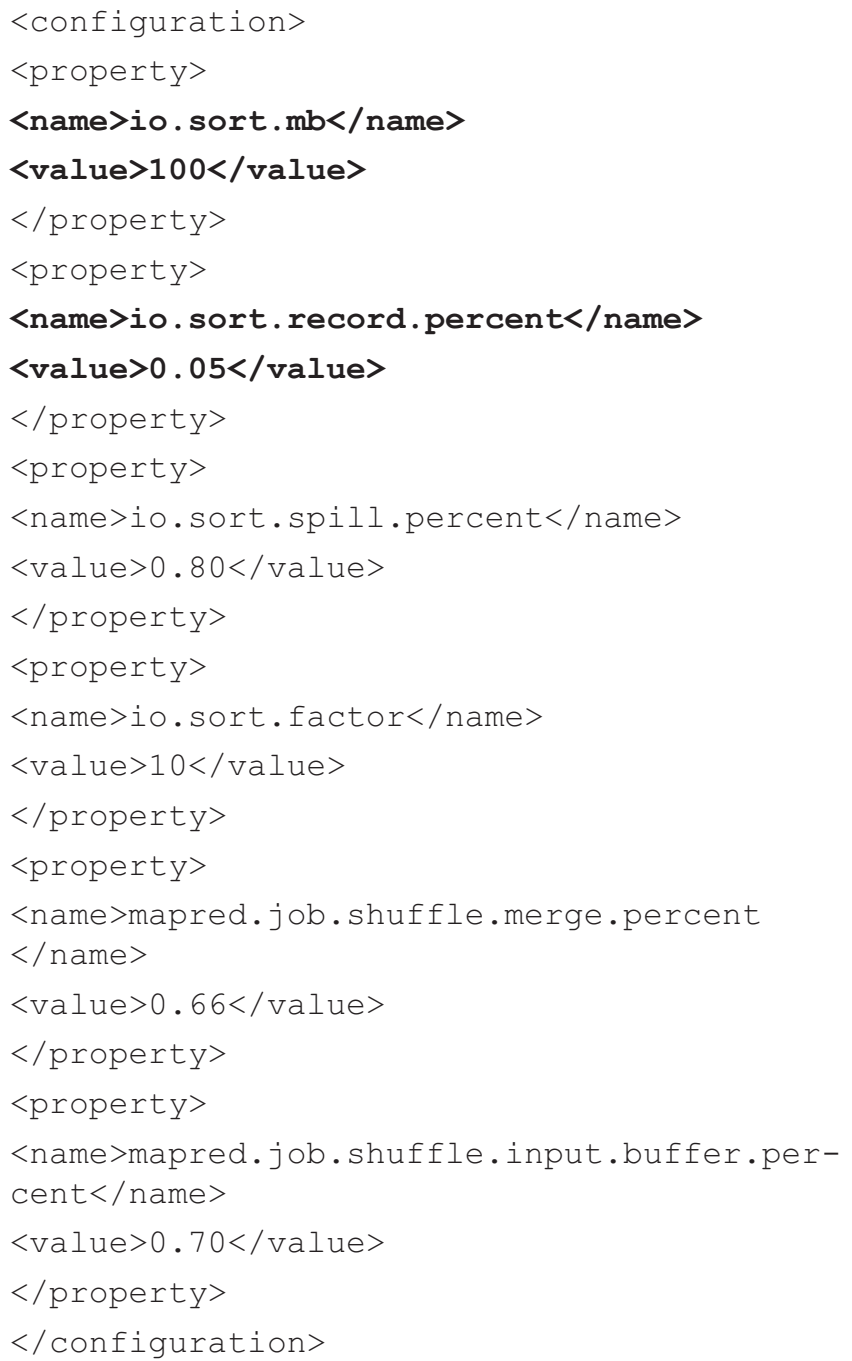

There are several steps to set up parameter values static way, first stop all the processes (Hadoop SecondaryNameNode, DataNode, TaskTracker, JobTracker, NameNode) on the cluster using stopall.sh, manually modify the configuration, and then use start-all. sh to start all the processes. Apache Hadoop operates using the default value defined in \$hadoop_home/docs/hdfs-default.xml, if required parameters are not defined in the \$hadoop_home/conf/ mapred-site.xml.

At the previous work we have developed a java application framework to modify configuration files without human intervention and applied at the several parameter evaluations [9-12].

As shown in Table 1, we have selected six important parameters for evaluation set which includes a domain of double or integer type. Each parameter distinguishes the minimum value from the maximum value based on the default value. When the tuning is completed, one value will be determined for each parameter.

\subsection{Parameter Value Domains}

As shown in Table 2, all parameter values are constantly subdivided by ranging from $-90 \%$ up to $+90 \%$ based on its low and high values [11]. The reason for configuring this arity table is to broaden the selection that one parameter can have.

\subsection{Parameter Space}

The parameter space $[9,11]$, denoted by $\Omega(x)$ is a sample space composed of parameter combinations taken from arity table and its time difference generated by map and reduce applications.

Depending on the algorithm chosen, the number of parameter combinations can be variably changed. If we apply Naïve exhaustive algorithm, the size of the space will hold $a^{\wedge} p$ cases with its evaluation results (where $a$ : arity, $n$ : number of parameter).

While the heuristically greedy algorithm requires multiple $\Omega(x)$ spaces which will hold each cases respectively, e.g., $a^{*} p, a^{\star}(p-1)$, $a^{*}(p-2), \ldots, a^{*}(p-(p-1))$.

\subsection{Benchmarking Applications}

Apache Hadoop distribution provide predeveloped several stress testing tools such as TestDFSIO, TeraSort, MrBench and MnBench in forms of hadoop-test-1.2.1.jar or hadoopexamples-1.2.1.jar.

Table $1 \mid$ Parameters and its values

\begin{tabular}{llccc}
\hline Symbol & Parameter and description & Default & Low & High \\
\hline P0(i.s.m) & io.sort.mb & 100 & 50 & 120 \\
P1(i.s.r.p) & io.sort.record.percent & 0.05 & 0.01 & 0.09 \\
P2(i.s.s.p) & io.sort.spill.percent & 0.80 & 0.40 & 0.90 \\
P3(i.s.f) & io.sort.factor & 10 & 1 & 20 \\
P4(m.j.s.m.p) & $\begin{array}{l}\text { mapred.job.shuffle.merge. } \\
\text { percent }\end{array}$ & 0.66 & 0.44 & 0.88 \\
P5(m.j.s.i.b.p) & $\begin{array}{l}\text { mapred.job.shuffle.input. } \\
\text { buffer.percent }\end{array}$ & 0.70 & 0.40 & 0.90 \\
\hline
\end{tabular}


Table 2 Arity table of parameters

\begin{tabular}{|c|c|c|c|c|c|c|c|c|c|}
\hline Par $\backslash$ Arity\% & $-90 \%$ & $-80 \%$ & $-70 \%$ & $-60 \%$ & $-50 \%$ & $-40 \%$ & $-30 \%$ & $-20 \%$ & $-10 \%$ \\
\hline i.s.m & 50 & 55 & 61 & 66 & 72 & 77 & 83 & 88 & 94 \\
\hline i.s.r.p & 0.01 & 0.014 & 0.019 & 0.023 & 0.028 & 0.032 & 0.037 & 0.041 & 0.046 \\
\hline i.s.s.p & 0.4 & 0.444 & 0.489 & 0.533 & 0.578 & 0.622 & 0.667 & 0.711 & 0.756 \\
\hline i.s.f & 1 & 2 & 3 & 4 & 5 & 6 & 7 & 8 & 9 \\
\hline m.j.s.m.p & 0.22 & 0.269 & 0.318 & 0.367 & 0.416 & 0.464 & 0.513 & 0.562 & 0.611 \\
\hline m.j.s.i.b.p & 0.2 & 0.256 & 0.311 & 0.367 & 0.422 & 0.478 & 0.533 & 0.589 & 0.644 \\
\hline Par $\backslash$ Arity\% & $+10 \%$ & $+20 \%$ & $+30 \%$ & $+40 \%$ & $+50 \%$ & $+60 \%$ & $+70 \%$ & $+80 \%$ & $+90 \%$ \\
\hline i.s.m & 111 & 122 & 133 & 144 & 155 & 166 & 177 & 188 & 200 \\
\hline i.s.r.p & 0.056 & 0.061 & 0.067 & 0.072 & 0.078 & 0.083 & 0.089 & 0.094 & 0.1 \\
\hline i.s.s.p & 0.811 & 0.822 & 0.833 & 0.844 & 0.856 & 0.867 & 0.878 & 0.889 & 0.9 \\
\hline i.s.f & 11 & 12 & 13 & 14 & 15 & 16 & 17 & 18 & 20 \\
\hline m.j.s.m.p & 0.684 & 0.709 & 0.733 & 0.758 & 0.782 & 0.807 & 0.831 & 0.856 & 0.88 \\
\hline m.j.s.i.b.p & 0.722 & 0.744 & 0.767 & 0.789 & 0.811 & 0.833 & 0.856 & 0.878 & 0.9 \\
\hline
\end{tabular}

The TestDFSIO [12] for measuring throughput, average IO rate $\mathrm{mb} / \mathrm{s}$, IO rate std deviation, and execution time on the HDFS clusters are as follows:

(e.g. -nrFiles 10, -fileSize 100)

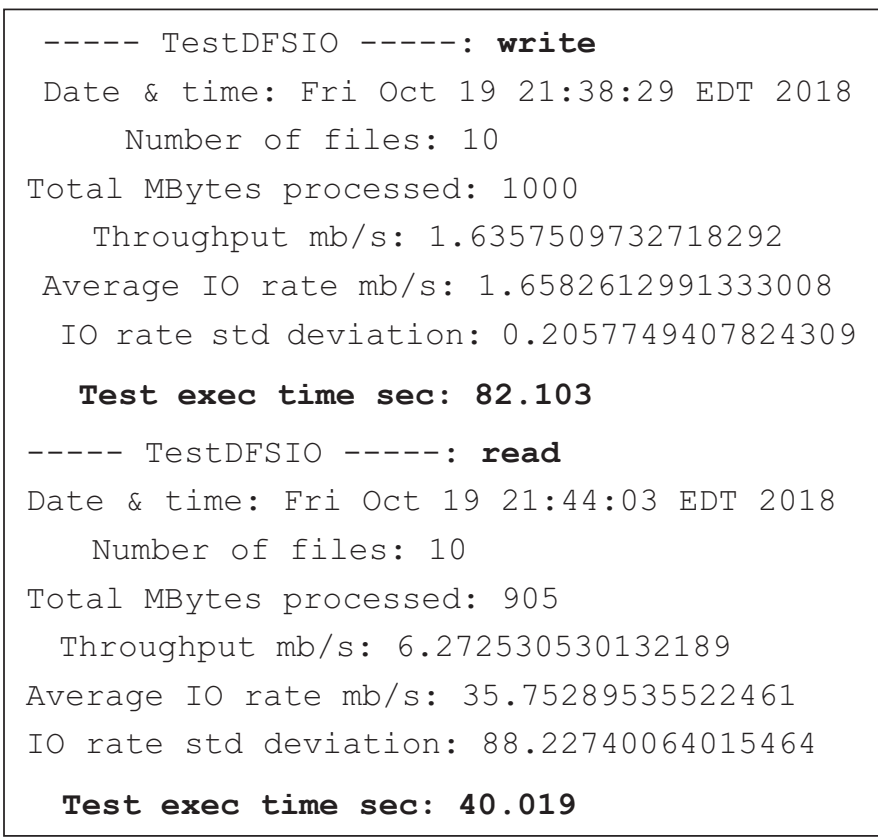

The TeraSort [12] is a benchmark that combines testing the HDFS and MapReduce of a Hadoop cluster [15]. It provides MapReduce framework, File system counters, and Job Counters.

\begin{tabular}{|l|}
\hline---- Tera Sort: $($ sort $30 \mathrm{~K}$ ) \\
Map input bytes $=30000$ \\
Reduce input groups $=300$ \\
Combine output records $=0$ \\
Reduce output records $=300$ \\
Map output records $=300$ \\
CPU time spent (ms) $=850$
\end{tabular}

The MrBench [12] is an application to measure performance by executing several small-scale jobs multiple times, as opposed to large tasks on TeraSort. Following example shows average time consumption for 30 runs.

\$ hadoop jar hadoop-*test ${ }^{*}$.jar mrbench -numRuns 30

$\begin{array}{cccc}\text { DataLines } & \text { Maps } & \text { Reduces } & \text { AvgTime (ms) } \\ 1 & 2 & 1 & \mathbf{3 1 4 1 4}\end{array}$

\subsection{Cost of the Parameter Selection Algorithms}

The execution time of map and reduce task will not be estimated before evaluation process has finished. This is because the correlation between one value of parameter and the others does not have a linear relationship. To verify this, we have to create a combination of values and run each case in the actual Hadoop environment. If it takes too much time, benchmarking will be less effective.

Considering three methods we have proposed, we can express the execution time as a Big $\mathrm{O}$ notation as follows:

\subsubsection{Cost comparison}

$$
\begin{aligned}
& >\text { Naïve exhaustive: } O\left(a^{\wedge} p\right) \\
& >\text { Random method: } O\left(1^{\star} p{ }^{\star} r\right) \approx O\left(P^{\wedge} 2\right) \\
& >\text { Greedy: } O\left(a^{\star}(p+(p-1)+(p-2)+\ldots p-(p-1))\right) \approx O\left(p^{\wedge} 3\right)
\end{aligned}
$$

Where, $p$ : number of parameters, 1 : constant time, $a$ : number of parameter values.

The execution time of Naïve exhaustive method can be expressed as $O\left(a^{\wedge} p\right)$ because you have to take into account the number of all cases, which in other words can be called the combinatorial problem. This approach is beneficial for finding appropriate parameters, but there is a time-consuming disadvantage. 
To compensate for these drawbacks, the random method proposed. In this method, a parameter value is picked from the arity table by generating random numbers ranging from zero to 17 . With this method, we could shorten the execution time, but we could not find a good candidate parameter compared to the Naïve exhaustive.

Finally, the heuristically greedy proposed to save benchmarking time and find acceptable parameters. An important point of this method was to fix the parameter value that most drastically reduced execution time in one parameter space and to test other parameters based on it.

Figure 1 shows how the benchmarking time changes as the number of parameters to be tested increases. Here we can see the heuristically greedy lies between Naïve and Random, but almost similar to Random's execution time.

It is difficult to determine a linear correlation between each parameter combination.

The heuristically greedy method is less efficient compared to the random [10], but it has been verified in previous studies as a computationally feasible way to find the best combination of parameters. The heuristically-based parametric optimization took slightly over quadratic time or at most cubic time and the performance is also great with feasible optimization time [11].

\section{3. e-HEURISTIC GREEDY MODEL}

The algorithms we proposed for extracting parameter sets is single benchmark application exercised to a one algorithm. For example, the TestDFSIO was applied to Naïve exhaustive and Random, the TeraSort was tested on the heuristically greedy.

However, in actual systems, it is also important to identify the parameter set that are applicable to common tasks because multiple map reduce operations are performed with different characteristics. So, we present the extended model called e-heuristic greedy that can be fulfilled for the multiple applications by extending heuristically greedy.

In this model, we evaluate map reduce performance with TeraSort, TestDFSIO, and MrBench on the Hadoop HDFS. Likewise, the heuristically-based [11] greedy, in the selection process of each stage assumes one parameter value contributing to the most significant performance reduction in the parameter space is selected to determine the subsequent parameters.

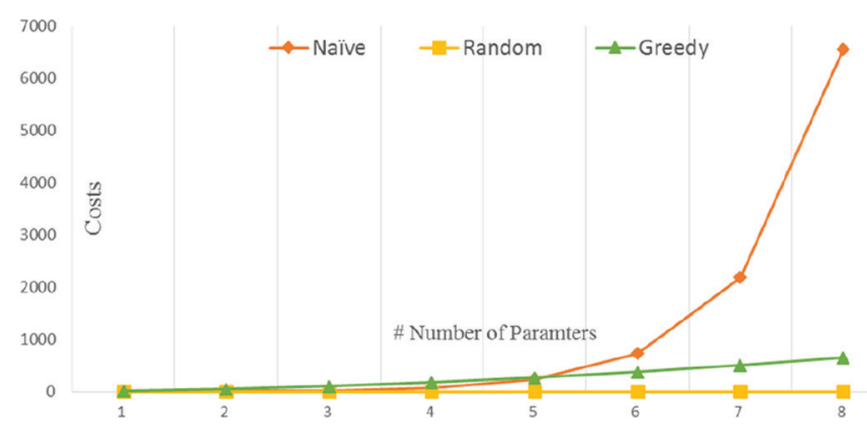

Figure 1 Optimization cost of e-Heuristic greedy

\section{1. e-Heuristic Greedy Structure and Operations}

The overall main logic of e-Heuristic greedy is similar to heuristically greedy, but in the e-heuristic greedy, two more functions are combined to hold MrBench and TestDFSIO benchmarks. This model takes the form of batch processing. Once it is executed, the Hadoop configuration is created and three benchmarking programs are sequentially executed based on this configuration. This is because Hadoop HDFS must be restarted to apply changed parameter values for the new task. The component of the parameter space that we set conceptually defined are stored in a single text file in the Linux system.

\section{2. e-Heuristic Greedy - Parameter Space}

The parameter evaluation space denoted by $\Omega$ composed of $n-1$ spaces from $\Omega(0), \Omega(1), \ldots, \Omega(n-1)$ where $n$ : number of configuration parameters. The $\Omega(0)$ is an initial evaluation space for all parameters which includes set of values of parameter and its evaluation results generated by benchmarking applications.

The first benchmarking uses the default parameter values (i.s.m 100, i.s.r.p 0.05, i.s.s.p 0.80, i.s.f 10, m.j.s.m.p 0.66, m.j.s.i.b.p 0.70) provided by Hadoop distribution. Based on this subsequent processing continues with this value and the result is stored in the $\Omega(x+1)$.

As shown in Table 3 , the $\Omega(x)$ space is composed of parameter values taken from the arity table and the execution time difference value of benchmarking compare to the result by generating Hadoop default parameter values. In order to find out how each parameter affects the execution result, 18 test cases are performed for each parameter while others are retained default value.

In Table 3 consider the fifth row, highlighted yellow, this is an example for the parameter $p 2$ indexed by column $3^{\text {rd }}$ which is i.s.s.p (io.sort.spill.percent).

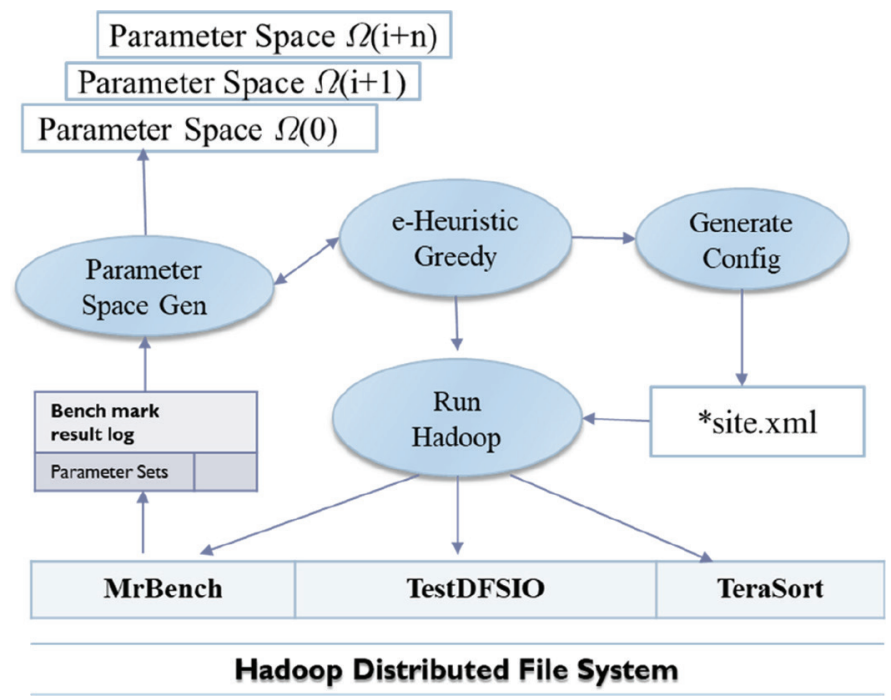

Figure 2 e-Heuristic greedy model 
Table $3 \mid \Omega(x)$ space components

\begin{tabular}{lccccccccr}
\hline Inc & $\mathbf{\%}$ & inx & $\boldsymbol{p 0}$ & $\boldsymbol{p 1}$ & $\boldsymbol{p} \mathbf{2}$ & $\boldsymbol{p 3}$ & $\boldsymbol{p 4}$ & $\boldsymbol{p} \mathbf{5}$ & Diff \\
\hline+ & 10 & 0 & 111 & 0.05 & 0.8 & 10 & 0.66 & 0.7 & 70 \\
+ & 30 & 4 & 100 & 0.05 & 0.8 & 10 & 0.733 & 0.7 & 620 \\
- & 30 & 5 & 100 & 0.05 & 0.8 & 10 & 0.66 & 0.533 & 1020 \\
+ & 90 & 2 & 100 & 0.05 & 0.9 & 10 & 0.66 & 0.7 & -2770 \\
- & 90 & 3 & 100 & 0.05 & 0.8 & 2 & 0.66 & 0.7 & 1270 \\
\hline
\end{tabular}

The i.s.s.p increases the default value of 0.8 by $+90 \%$ to obtain a value of 0.9 , and the remaining five parameters retain the default value $\{p 0$ : $100, p 1: 0.05, p 2: 0.9, p 3: 10, p 4: 0.66, p 5: 0.7\}$. After changing the Hadoop configuration file with the given one set of parameter combinations, $p 0-p 5$, three benchmarking programs are run under the same conditions.

The execution time difference, denoted by Diff, is calculated by comparing the result obtained on the combination of default parameter values and newly updated ones.

- Calculate run time differences

Diff $=($ diff TestDFSIO read + diff TestDFSIO write + diff TeraSort exec + diff TeraSort CPU + diff MrBench exec)

diff TestDFSIO read $=($ TestDFSIO read - default TestDFSIO read $)$

diff TestDFSIO write $=($ TestDFSIO read - default TestDFSIO write $)$

diff TeraSort exec $=($ TeraSort exec - default TeraSort $)$

diff TeraSort CPU $=($ TeraSort CPU - default CPU $)$

diff MrBench exec $=($ MrBench exec - default CPU $)$

\section{3. e-Heuristic Greedy Model - Determining Parameter}

This model also assumes the values that can be locally minimized the benchmarking results in the current stage will ultimately lead to a global optimum.

Before moving on to the next stage $\Omega(n+1)$ from the current $\Omega(n)$, the parameter and its corresponding value that played a significant role in reducing system performance need to be determined by evaluating Diff value.

The only one parameter and associated value is fixed for each stage, and in the next stage, the values in the attribute table are applied to each parameter to pin down. By doing this the parameter space will be eventually decreased.

- $\Omega(x)$ space reduction

$$
\Omega(0)=a^{\star} p, \Omega(1)=a^{\star}(p-1), \ldots, \Omega(n)=a^{\star}(p-n)
$$

where $p$ : number of parameters, $a$ : arity, $n$ : number of stages.

- Total cost of benchmarking

$$
\sum_{i=0}^{n}\left(\left(a^{\star}(p-i)\right)\right)
$$

Table 4 shows how parameters are fixed when there are six parameters. For example, if $p=6$ and $a=18$, the stage for tuning requires five $\Omega$ spaces, one additional parameter is fixed as the stage increases, and the remaining one parameter is determined in the final stage.

\section{4. e-Heuristic Greedy with Alternate Parameter Selection}

In the e-heuristic greedy model, the parameter is fixed based on the local minimum for each stage. However, if the local minimum value is less than previous stage, it is necessary to consider next possible candidate parameter from the previous stage and re-evaluate current stage with newly selected one to expect leading better performance.

Figure 3 shows the processing steps and its evaluation costs. Each bubble indicates the state that exists in the evaluation spaces and arrows show the progression direction between the stages.

For example, in Case 2, the selected Diff value is larger than a state $\mathrm{S} 1$ in $\Omega(1)$ compare to the state $\mathrm{S} 0$ in $\Omega(0)$. In this case, after selecting the candidate parameter and its values which is second smallest Diff from S0 and the S1 is re-evaluated with the newly updated sets and the result is saved at S6.

This methodology, each state can be performed once more, so the following additional cost is required. However, we expect to find better results than in e-heuristic greedy, and the performance test for this model will proceed in the next study.

\begin{tabular}{|c|c|c|c|c|c|}
\hline$\Omega(0)$ & $\Omega(1)$ & $\Omega(2)$ & $\Omega(3)$ & $\Omega(4)$ & $\Omega(5)$ \\
\hline${ }^{*}$ i.s.m & ${ }^{*}$ i.s.m & ${ }^{*}$ i.s.m & ${ }^{*}$ i.s.m & ${ }^{\star}$ i.s.m & ${ }^{\star}$ i.s.m \\
\hline i.s.r.p & ${ }^{*}$ i.s.r.p & ${ }^{*}$ i.s.r.p & ${ }^{*}$ i.s.r.p & ${ }^{*}$ i.s.r.p & ${ }^{*}$ i.s.r.p \\
\hline i.s.r.p & i.s.r.p & ${ }^{*}$ i.s.s.p & ${ }^{*}$ i.s.s.p & ${ }^{*}$ i.s.s.p & ${ }^{*}$ i.s.s.p \\
\hline i.s.f & i.s.f & i.s.f & ${ }^{*}$ i.s.f & ${ }^{*}$ i.s.f & ${ }^{*}$ i.s.f \\
\hline m.j.s.m.p & m.j.s.m.p & m.j.s.m.p & m.j.s.m.p & ${ }^{*}$ m.j.s.m.p & ${ }^{\star}$ m.j.s.m.p \\
\hline m.j.s.i.b.p & m.j.s.i.b.p & m.j.s.i.b.p & m.j.s.i.b.p & m.j.s.i.b.p & ${ }^{*}$ m.j.s.i.b.p \\
\hline
\end{tabular}

Table 4 Parameter fixed for each space

\begin{tabular}{|c|c|c|c|c|c|c|}
\hline Cases $^{\Omega(x)}$ & $\Omega(0)$ & $\Omega(1)$ & $\Omega(2)$ & $\Omega(3)$ & $\Omega(4)$ & $\Omega(5)$ \\
\hline$I$ & & & & & & \\
\hline 2 & so, s1 & & & & & \\
\hline 3 & so, s1 & S6, S7 & & & & \\
\hline 4 & so, s1 & \$6, S7 & s11, s12 & & & \\
\hline 5 & so, s1 & S6, S7 & s11, s12 & s15, S16 & & \\
\hline 6 & so, s1 & S6, S7 & $\mathrm{s} 11, \mathrm{~s} 12$ & s15, s16 & s18, s19 & \\
\hline
\end{tabular}

"Parameters determined for the each $\Omega(x)$

Figure 3 Parameter fixed for each space 


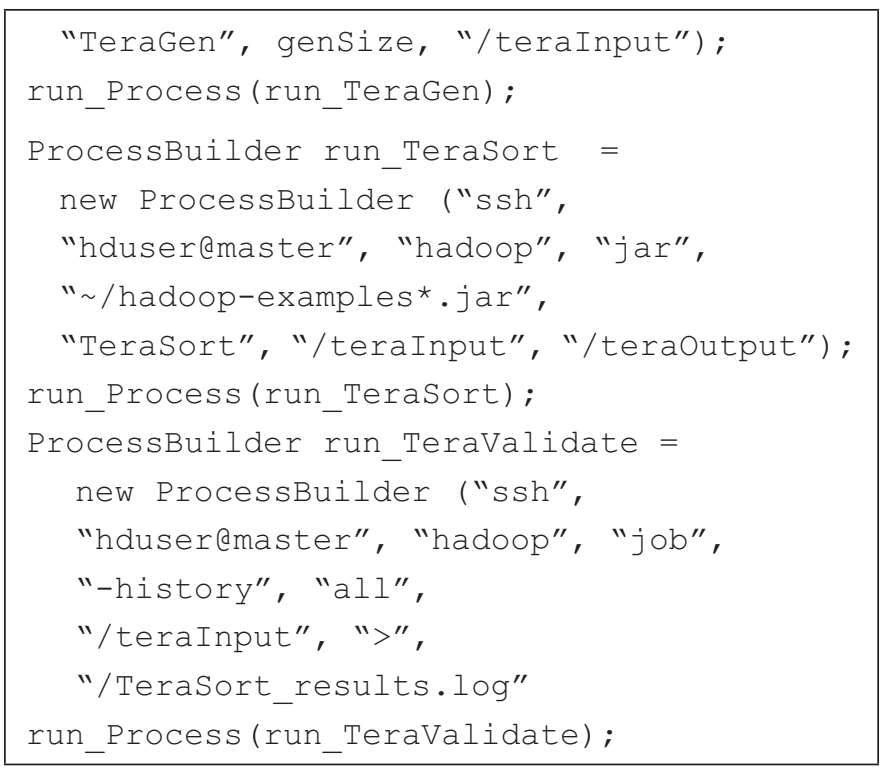

\subsubsection{MrBench}

MrBench is also implemented in the same way as TestDFSIO. In this way, for the benchmarking, it is possible to substitute the manual typing on the command line of the Linux OS by the process builder in succession.

Algorithm 4. Function runMrBench

Function: runMrBench
Input: processDir, numRuns
Output: Execution time
ProcessBuilder run_MrBench =
new ProcessBuilder ("hadoop", "jar",
" / hadoop-*test*.jar", "mrbench",
"-baseDir",
processDir, "-numRuns", numRuns);
run_Process (run_MrBench);
run_Process (ProcessBuilder pb) ;
Process process = pb.start ( );
\}

\section{EVALUATION AND ANALYSIS}

In this study, one Hadoop cluster consist of one master node and four slave nodes are used. Master node is configured with Intel(R) Core(TM) i5-6500 CPU, 3.20 GHz and $8 \mathrm{~GB}$ memory; and slave nodes with Intel(R) Core(TM) i3-6100T CPU, 3.20 GHz, and 8 GB memory. Each machine is bundled via $100 \mathrm{Mbps}$ SAU Local Area Network. Hadoop version 1.2.1 and CentOS Linux release 7.3.1611 (Core) is used for the evaluation.

As shown in Table 5, a master node includes DataNode, NameNode, SecondaryNameNode, TaskTracker, JobTracker, on each slave node has TaskTracker and DataNode.

\subsection{Evaluation Results}

The performance of HDFS and MapReduce workloads are measured by TeraSort with Gensize $30 \mathrm{MB}$, MrBench with five runs, and TestDFSIO with filesize $20 \mathrm{MB}$. As shown in the following steps, one parameter and its associated values are fixed in each evaluation space. For the display purpose, the Diff value is multiplied by 10 to visualize on the graph.

\subsubsection{Default parameter evaluation}

To set up the reference value of the parameter tuning, default values are applied to the six selected parameters. The total execution time of $58.01 \mathrm{~s}$ is set as the reference value.

- Exec time: $58.01 \mathrm{~s}$

- Default: \{i.s.m: (100), i.s.r.p: (0.05), i.s.s.p: (0.80), i.s.f: (10), m.j.s.m.p: (0.66), m.j.s.i.b.p: (0.70)\}

\subsubsection{Parameter evaluation space $\Omega(0)$}

In this parameter space $\Omega(0)$, the parameter that has the greatest effect on the total execution time is fixed to m.j.s.m.p. with its value 0.709 .

- Id $x: 4$, m.j.s.m.p (mapred.job.shuffle.merge.percent)

- Increased +20\%: 0.709, Exec: 45.69, Diff: $-12.32 \mathrm{~s}$

Table 5 Processors on Hadoop clusters

\begin{tabular}{llll}
\hline Nodes & Master node & Slaves 1 & Slaves 2 \\
\hline IP Address & 10.106.*.203 & 10.106..$^{*} 22$ & $10.106 .^{*} .136$ \\
Processors & 6931 TaskTracker & 6928 TaskTracker & 12844 TaskTracker \\
on nodes & 7043 RunJar & 6795 DataNode & 12735 DataNode \\
& 6744 JobTracker & Slaves 3 & Slaves 4 \\
& 7208 RunJar & 10.106..$^{*}$.164 & 10.106. .163 \\
& 6345 DataNode & 13040 TaskTracker & 3795 TaskTracker \\
& 6571 S-NameNode & 12935 DataNode & 3659 DataNode \\
& 6143 NameNode & & \\
\hline
\end{tabular}

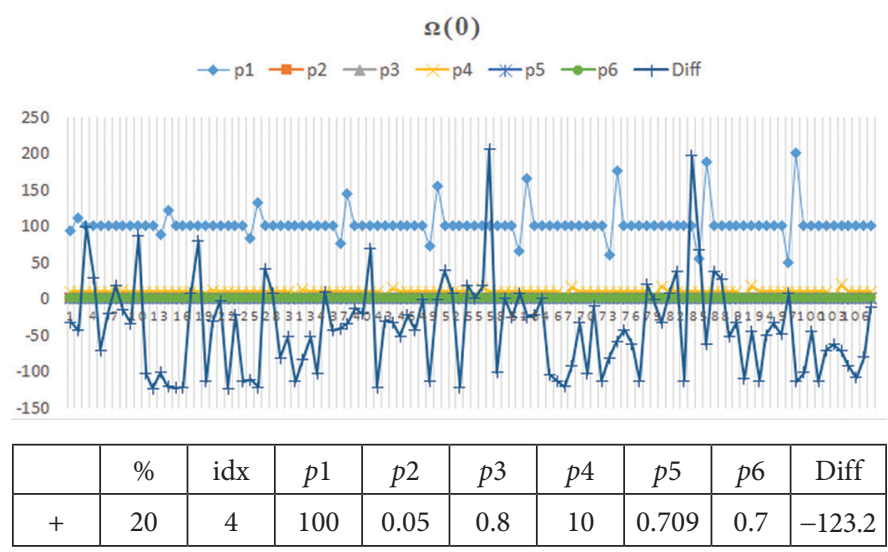

Figure 4 Parameter trends on $\Omega(0)$ 
- Fixed: \{m.j.s.m.p: (0.709)\}

- Default: \{i.s.m: (100), i.s.r.p: (0.05), i.s.s.p: (0.80), i.s.f: (10), m.j.s.i.b.p: $(0.70)\}$

\subsubsection{Parameter evaluation space $\Omega(1)$}

In $\Omega(1)$, the i.s.r.p with 0.032 is selected as the second candidate. We have here established two parameters m.j.s.m.p and i.s.r.p.

- $\operatorname{Id} x: 1$, i.s.r.p (io.sort.record.percent)

- Increased -40\%: 0.032, Exec: 43.91, Diff: $-14.31 \mathrm{~s}$

- Fixed: \{m.j.s.m.p: (0.709), i.s.r.p: (0.032)\}

- Default: \{i.s.m: (100), i.s.s.p: (0.80), i.s.f: (10), m.j.s.i.b.p: (0.70)\}

\subsubsection{Parameter evaluation space $\Omega(2)$}

- Id $x$ : 3, i.s.f (io.sort.factor)

- Increased +60\%: 16 , Exec: 41.81 , Diff: $-16.2 \mathrm{~s}$

- Fixed: \{m.j.s.m.p: (0.709), i.s.r.p: (0.032), i.s.f: (16)\}

- Default: \{i.s.m: (100), i.s.s.p: (0.80), m.j.s.i.b.p: (0.70)\}

\subsubsection{Parameter evaluation space $\Omega(3)$}

- Id $x$ : 2, i.s.s.p (io.sort.spill.percent)

- Increased +10\%: 0.811, Exec: 42.91, Diff: $-15.1 \mathrm{~s}$

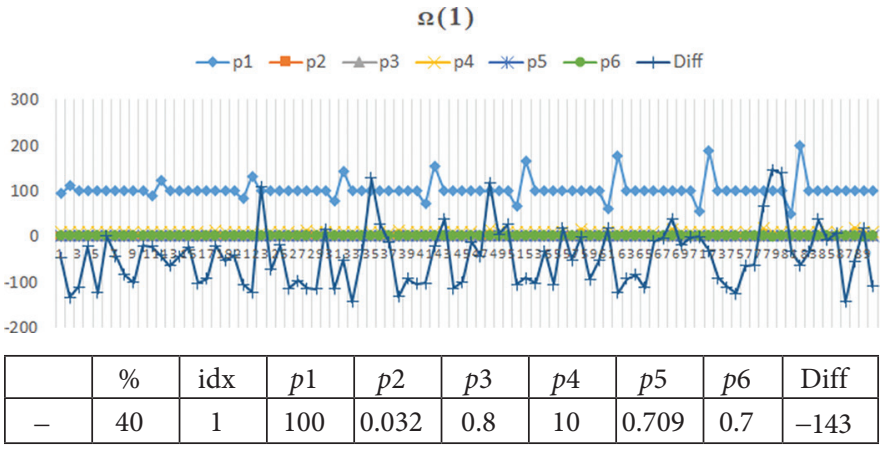

Figure 5 Parameter trends on $\Omega(1)$

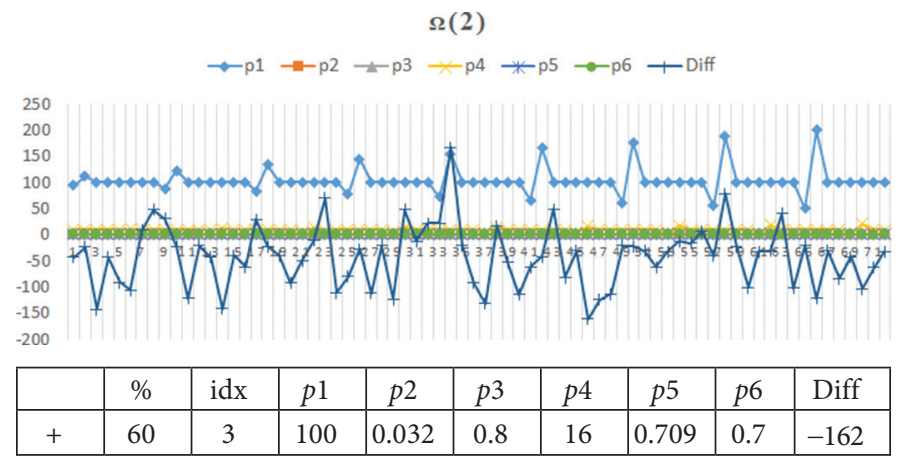

Figure 6 Parameter trends on $\Omega(2)$
- Fixed: \{m.j.s.m.p: (0.709), i.s.r.p: (0.032), i.s.f: (16), i.s.s.p: $(0.811)\}$

- Default: $\{$ i.s.m: (100), m.j.s.i.b.p: $(0.70)\}$

\subsubsection{Parameter evaluation space $\Omega(4)$}

- $\operatorname{Id} x$ : 0, i.s.m (io.sort.mb)

- Increased -60\%: 66, Exec: 40.81, Diff: $-17.2 \mathrm{~s}$

- Fixed: \{m.j.s.m.p: (0.709), i.s.r.p: (0.032), i.s.f: (16), i.s.s.p: (0.811), i.s.m: $(66)\}$

- Default: $\{$ m.j.s.i.b.p: $(0.70)\}$

\subsubsection{Parameter evaluation space $\Omega(5)$}

- Id $x: 5$, m.j.s.i.b.p (mapred.job.shuffle.input.buffer.percent)

- Increased -10\%: 0.533, Exec: 42.81, Diff: $-15.2 \mathrm{~s}$

- Fixed: \{m.j.s.m.p: (0.709), i.s.r.p: (0.032), i.s.f: (16), i.s.s.p: (0.811), i.s.m: (66), m.j.s.i.b.p: $(0.533)\}$

- Default: \{\}

\subsection{Total Benchmarking Space Trend Analysis}

Through the e-heuristic greedy algorithm, parameters are identified for each evaluation space as shown in Table 6. We did not find the most optimal parameter set in $\Omega(5)$ at the last stage, but fortunately we could identify the set that optimizes performance

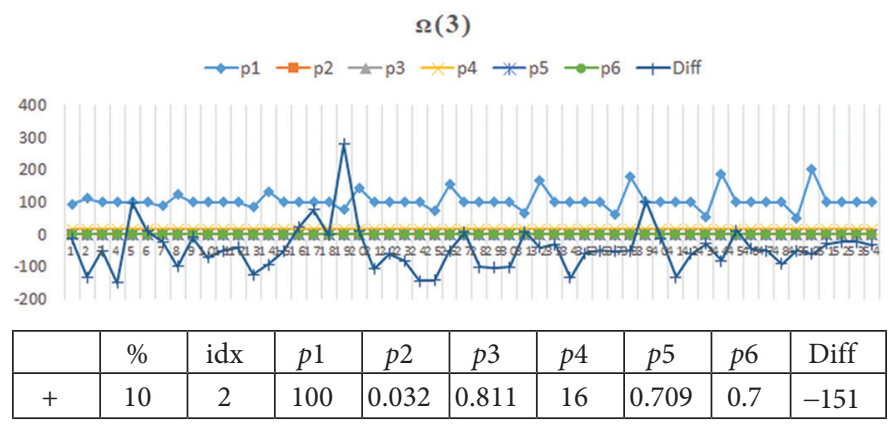

Figure 7 Parameter trends on $\Omega(3)$

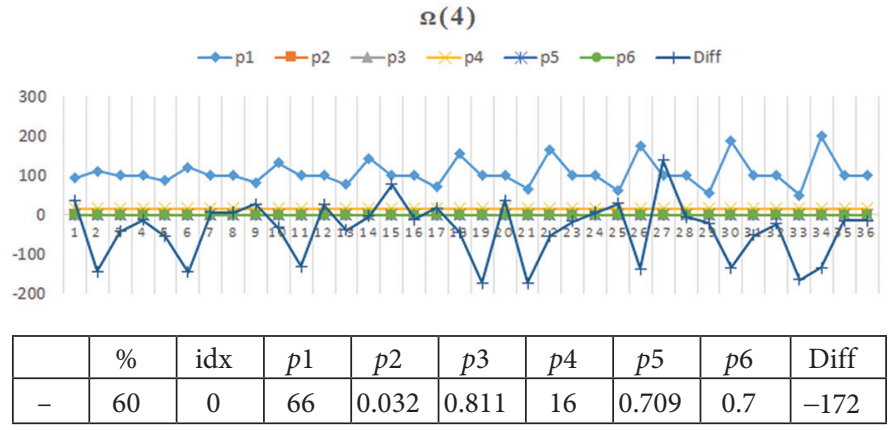

Figure 8 Parameter trends on $\Omega(4)$ 


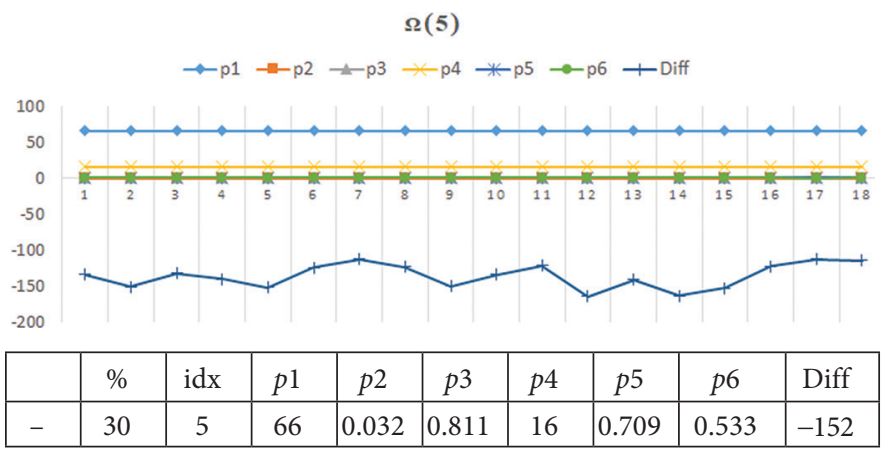

Figure 9 Parameter trends on $\Omega(5)$

Table 6 Total parameter trend on all spaces

\begin{tabular}{lcclcccc}
\hline $\boldsymbol{\Omega} \backslash \boldsymbol{p}$ & $\boldsymbol{p} \mathbf{1}$ & $\mathbf{p} \mathbf{2}$ & $\mathbf{p 3}$ & $\mathbf{p 4}$ & $\mathbf{p 5}$ & $\mathbf{p 6}$ & Diff \\
\hline$\Omega(0)$ & 100 & 0.05 & 0.8 & 10 & 0.709 & 0.7 & -12.32 \\
$\Omega(1)$ & 100 & 0.032 & 0.8 & 10 & 0.709 & 0.7 & -14.31 \\
$\Omega(2)$ & 100 & 0.032 & 0.8 & 16 & 0.709 & 0.7 & -16.18 \\
$\Omega(3)$ & 100 & 0.032 & 0.811 & 16 & 0.709 & 0.7 & -15.11 \\
$\Omega(4)$ & 66 & 0.032 & 0.811 & 16 & 0.709 & 0.7 & -17.23 \\
$\Omega(5)$ & 66 & 0.032 & 0.811 & 16 & 0.709 & 0.533 & -15.23 \\
\hline
\end{tabular}

among the six spaces in $\Omega(4)$ with a parameter set and values \{m.j.s.m.p: (0.709), i.s.r.p: (0.032), i.s.f: (16), i.s.s.p: (0.811), i.s.m: (66), m.j.s.i.b.p: $(0.70)\}$ to minimize execution time of -17.2 s. It can be said that the local minimum value selected at each stage contributed to finding a globally optimal value.

\section{CONCLUSION}

This paper has presented a method to facilitate the extraction of common parameter set for a Hadoop cluster by applying multiple benchmarking application include TeraSort, TestDFSIO and MrBench.

This model developed by extending the heuristically-based greedy to adapt more complex map and reduce tasks. In each parameter evaluation space, one parameter and its associated value is selected that is considered to be the most locally optimum value by considering total sum of execution time. Since we assume that the selected value will ultimately lead to a global optimum, the value of this parameter is fixed in the next evaluation space and the remaining available parameters are tested.

As a result of selecting sequence, this model has found one set of parameter that shortens the execution time by $27 \%$ compared with default one at the evaluation space $\Omega(4)$. In each space, of course we could classify the parameter combinations with better processing time than the default one, but we found that it may not be possible to find better combinations as move on to the next space.

\section{ACKNOWLEDGEMENT}

This study is supported by the FY1718 faculty research grant from Southern Arkansas University (10-2860-5-020, 10-2860-6180).

\section{REFERENCES}

[1] Apache Hadoop Home Page, http://hadoop.apache.org/ (Last accessed - Dec 20, 2017).

[2] H. Han, Y. Wen, T.-S. Chua, X. Li, Toward scalable systems for big data analytics: a technology tutorial, in: IEEE Access, Vol. 2, IEEE, 24 June 2014, pp. 652-687 (Big Data Framework).

[3] S. Ghemawat, H. Gobioff, S.-T. Leung, The Google file system, in: Proceedings of the 19th ACM symposium on Operating systems principles, Vol. 37, ACM, New York, NY, USA, 19-22 October 2003, pp. 29-43.

[4] S. Babu, Towards automatic optimization of MapReduce programs, in: Proceedings of the 1st ACM symposium on Cloud computing, ACM, New York, NY, USA, 10-11 June 2010, pp. 137-142.

[5] J. Dean, S. Ghemawat, MapReduce: simplified data processing on large clusters, Communications of the ACM - 50th anniversary issue, ACM, New York, NY, USA, Vol. 51, January 2008, pp. 107-113.

[6] Technical White Paper, Performance measurement of a Hadoop cluster, AMAX, 2012.

[7] M. Kontagora, H. González-Vélez, Benchmarking a MapReduce environment on a full virtualization platform, in: 2010 International Conference on Complex, Intelligent and Software Intensive Systems (CISIS), IEEE, Krakow, 2010.

[8] The Fresh Open Source Software Archive, http://fossies.org/linux/ misc/hadoop-1.2.1.tar.gz/hadoop-1.2.1/docs/mapred-default. html. Web. 20 January 2015.

[9] J. Kim, T.K. Ashwin Kumar, K.M. George, N. Park, Performance evaluation and tuning for MapReduce computing in Hadoop distributed file system, in: 2015 IEEE 13th International Conference on Industrial Informatics (INDIN), IEEE, Cambridge, UK, 22-24 July 2015.

[10] J. Kim, N. Park, Identification of the optimal Hadoop configuration parameter sets for MapReduce computing, in: 2015 3rd International Conference on Applied Computing and Information Technology/2nd International Conference on Computational Science and Intelligence, IEEE, Okayama, 12-16 July 2015.

[11] J. Kim, N.-J. Park, N. Park, A study of heuristically-based parametric performance improvement/optimization algorithms for bigdata computing, in: 20164th Intl Conf on Applied Computing and Information Technology/3rd Intl Conf on Computational Science/Intelligence and Applied Informatics/1st Intl Conf on Big Data, Cloud Computing, Data Science \& Engineering (ACIT-CSII-BCD), IEEE, Las Vegas, NV, USA, 12-14 December 2016.

[12] M.G. Noll, Benchmarking and stress testing a Hadoop cluster with TeraSort, TestDFSIO \& co., http://www.michael-noll.com/blog/ 2011/04/09/benchmarking-and-stress-testing-an-hadoopcluster-with-terasort-testdfsio-nnbench-mrbench, 2011.

[13] T. White, Hadoop: The Definitive Guide, O'Reilly Media, Inc., 2011, pp. 177-178.

[14] Hadoop 1.2.1 Documentation, Mapred-default Configuration Parameters, https://hadoop.apache.org/docs/r1.2.1/mapred-default. html. Web. 20 January 2015.

[15] TeraSort Benchmark Comparison for YARN, TeraSort Benchmark Comparison for YARN | MapR, https://mapr.com/resources/terasort-benchmark-comparison-yarn/.

[16] ProcessBuilder (Java Platform SE 7), 23 June 2018, https://docs. oracle.com/javase/7/docs/api/java/lang/ProcessBuilder.html. 